\title{
In Vivo Evaluation of Functionalized Biomimetic Hydroxyapatite for Local Delivery of Active Agents
}

\author{
Johan Forsgren $^{1}$, Ulrika Brohede ${ }^{2}$, Sonya Piskounova ${ }^{3}$, Albert Mihranyan ${ }^{1}$, Sune Larsson ${ }^{4}$, \\ Maria Strømme ${ }^{1}$, Håkan Engqvist ${ }^{1}$
}

\footnotetext{
${ }^{1}$ Department of Engineering Sciences, Uppsala University, Uppsala, Sweden; ${ }^{2}$ Sandvik AB, Stockholm, Sweden; ${ }^{3}$ Department of Materials Chemistry, Uppsala University, Uppsala, Sweden; ${ }^{4}$ Department of Orthopaedics, Uppsala University Hospital, Uppsala, Sweden.

E-mail: hakan.engqvist@angstrom.uu.se
}

Received November $3^{\text {rd }}, 2010$; revised January $19^{\text {th }}, 2011$; accepted January $22^{\text {nd }}, 2011$.

\begin{abstract}
This study was carried out to investigate the biological response in vivo to biomimetic hydroxyapatite implant coatings functionalized with bisphosphonates and bone morphogenetic proteins. The functionalization was carried out by a simple soaking procedure in the operating room immediately prior to surgery. Cylindrical titanium samples with and without coatings were implanted in the distal femoral epiphysis of sheep and retrieved after 6 weeks. The histological analysis proved that all samples were integrated well in the tissue with no signs of intolerance. Fewer osteoclasts were observed in the vicinity of bisphosphonate-functionalized samples and the bone was denser around these samples compared to the other samples. Samples functionalized with bone morphogenetic protein induced more bone/implant contact but showed a more inconsistent outcome with reduced bone density around the samples. This study demonstrates a simple method to functionalize implant coatings, which provides surgeons with an option of patient-specific functionalization of implants. The observed biological impact due to the delivery of active molecules from the coatings suggests that this strategy may also be employed to deliver antibiotics from similar coatings.
\end{abstract}

Keywords: Implant, Titanium, Hydroxyapatite, Bisphosphonate, Bone Morphogenetic Protein-2

\section{Introduction}

The success rate of hip and knee arthroplasties is high; more than $90 \%$ of the patients are predicted to live more than 10 years with their implant before revision surgery is needed [1,2]. But despite the high success rate, the number of revision surgeries is still far from satisfactory. Only in the U.S., 36,000 revision total hip arthroplasties and 32,700 revision total knee arthroplasties were performed in 2003, and the annual number is expected to increase steadily [3]. Revision surgeries due to implant failure are often complicated, expensive and painful for afflicted patients. In a study from 1995 it was shown that patients undergoing revision orthopedic surgery need to stay longer at the hospital and the time in the operating room is significantly higher compared with primary surgery [4]. In two retrospect studies of revision hip and knee arthroplasties, as much as $39 \%$ of the hip surgeries [1] and $63 \%$ of the knee surgeries [5] were performed within 5 years following primary surgery. Infection and aseptic loosening are two common indications for early implant failure of uncemented prostheses [1,2]. Surgical site infections account for $38 \%$ of all nosocomial infections in the US [6] and the incidence rate can be as high as $10 \%$ at spinal surgery [7]. In a recent retrospect study of 9245 patients undergoing primary hip or knee arthroplasty, $65 \%$ of the developed infections appeared within one year after primary surgery [8]. These findings show that perioperative infections associated with the surgical wound is a challenge that needs to be addressed if the number of revision surgeries is to be reduced.

The interface between prosthetic implant and tissue is known to be a region of local immune depression with reduced resistance to microbes, often referred to as an immune-incompetent fibro-inflammatory zone [9]. In addition, implant migration due instability in the interface between bone and implant can damage the tissues 
and almost completely deplete the immune defenses [10]. Thus, these peri-prosthetic regions are highly sensitive for bacteria colonization and biofilm formation. To reduce the risk for bacteria colonization with consecutive development of infection, it is desirable with bactericidal effect on the implant surface and a firm fixation of the prosthesis. This could be obtained by local and sustained release of active agents from the implant surface that promotes more dense bone formation around the prosthesis and/or has a bactericidal effect. It has been shown that the number of early failures associated with poor implant fixation have been reduced when osteoconductive coatings have been deposited on the implant surfaces [11,12]. Hydroxyapatite, a calcium phosphate material resembling the mineral phase found in bone [13], is the most studied and applied osteoconductive material, and earlier in vitro studies have proven the feasibility of biomimetic hydroxyapatite (BHA) as carrier of various active agents such as antibiotics [14], bisphosphonates (BIS) [15] and bone morphogenetic proteins (BMP) [16] for targeted administration at the site of implant. In the present study, the possibility of incorporating various agents to a BHA coating by a rapid on-site loading method was evaluated in vivo. The aim is to provide surgeons with an option to choose on-site if and what type of drug should be incorporated into the coating. In the design of the study, BIS and BMP were chosen, as they affect the bone formation surrounding the implant, which easily can be analyzed. The effect of antibiotics would be on the inhibition of bacteria entering the incision wound and such study would be more complicated to perform and more difficult to defend from an ethical standpoint as it would involve a substantial risk for the animals.

\section{Materials and Methods}

\subsection{Implant Preparations}

Cylindrical implants of titanium grade 2 with $8.0 \mathrm{~mm}$ length and $3.5 \mathrm{~mm}$ diameter were used in this comparative study, see Figure 1. The implants were delivered with a mounting device used to screw the implants in the right position, this part was subsequently removed after fixation of the implants. Three different surface modifications of the implants were evaluated and compared to a native titanium surface, see Table 1. Out of the total 20 implants that were prepared, 5 were left non-coated and 15 were coated with a layer of BHA. Preparation of the BHA coatings was performed as descried in earlier work [17]. Briefly, a layer of anatase $\mathrm{TiO}_{2}$ was deposited on the titanium cylinders using physical vapor deposition (PVD) before the samples were immersed in $40 \mathrm{ml}$ Dulbecco's phosphate buffered saline (Dulbecco's PBS, Sigma-Aldrich) each for 4 days at $60^{\circ} \mathrm{C}$. The samples were subsequently retrieved and analyzed with scanning

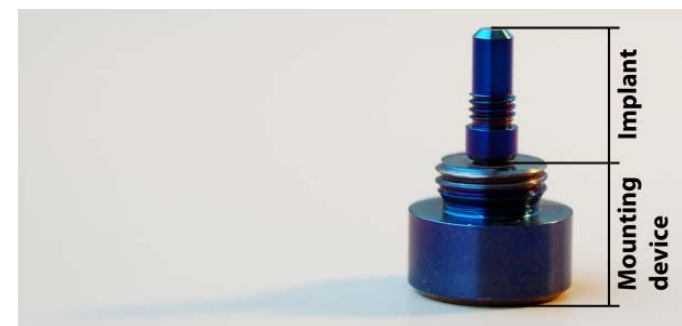

Figure 1. Photograph of implant where the cylindrical pin constitutes the actual sample, the screw at the top was used to fixate the implant in the cortical bone.

Table 1. Names and corresponding surface characteristics of the different sample.

\begin{tabular}{ccc}
\hline Sample type & No. of samples & Surface \\
\hline Control & 5 & Native titanium \\
Test 1 & 5 & BHA coating \\
Test 2 & 5 & BHA coating loaded with BMP-2 \\
Test 3 & 5 & BHA coating loaded with BIS \\
\hline
\end{tabular}

electron microscopy (SEM) to examine the precipitated layer of BHA. It was observed that the BHA-layer was not fully covering the cylinders and therefore the implants were once more immersed in PBS for additional 7 days at $37^{\circ} \mathrm{C}$. BHA-coatings prepared in a similar way have been described earlier [17-19] and have been shown to be highly porous and calcium deficient compared with stoichiometric HA, something that promotes incorporation of bioactive molecules [14,19]. After deposition of the BHA-layer, the implants were rinsed in deionized water, dried in air and sterilized by autoclaving (30 min, $120^{\circ} \mathrm{C}$ ). In the operating room, 5 BHA-coated implants were placed in individual sterile plastic tubes containing $0.5 \mathrm{ml}$ of an autoclaved aqueous solution of the bisphosphonatePamindronate $(0.5 \mathrm{mg} / \mathrm{ml}$, Sigma-Aldrich). Another 5 BHA-coated implants were soaked in a $0.5 \mathrm{ml}$ recombinant human BMP-2 solution (rhBMP-2, 0.15 $\mathrm{mg} / \mathrm{ml}$, Induct OS ${ }^{\circledR}$, Wyeth Europe) in formulation buffer containing $2.5 \%$ glycine, $0.5 \%$ sucrose, $0.01 \%$ Polysorbate $80,5 \mathrm{mM}$ sodium chloride and $5 \mathrm{mM} \mathrm{L}$-glutamic acid. The implants were immersed in the solutions for 10 - 30 minutes before implantation.

\subsection{Animal Study}

The in vivo study was designed for intra-osseous implantation in sheep for 6 weeks. Five 24-month-old female Grivette sheep, weighing from 51 to $63 \mathrm{~kg}$, were used in the study. Prior to the surgical procedure, the animals were fasted overnight. At the time of implantation, pre-medication and anesthesia was performed by intravenous injection of a thiopental-pentobarbital mixture (Nesdonal $^{\circledR}$, Merial; and Pentobarbital Sodique, CEVA Santé animale) and atropine (AtropinumSulfuricum, 
Aguettant) followed by the inhalation of an $\mathrm{O}_{2}$-isoflurane (1\% - 4\%) mixture (Afrane ${ }^{\circledR}$, Baxter). Each animal received a first analgesic (Flunixine, Meflosyl ${ }^{\circledR}$, Fort Dodge Santé Animale) and as a prophylactic measure, perioperative antibiotics penicillin procaine and penicillin benzathine (Duplocilline ${ }^{\circledR}$, Intervet) were given. In addition, each animal received a second analgesic (Buprenorphine, Temgesic ${ }^{\circledR}$, Schering Plough) preoperatively.

Bone defects were created bilaterally in the distal femoral epiphysis and the sites were implanted with samples. Each animal was implanted with 4 samples, one of each type. An orthopedic drill was used to create cylindrical defects with approximately $2.3 \mathrm{~mm}$ in diameter by $<9 \mathrm{~mm}$ deep in the distal femoral epiphysis. Drilling was performed under constant irrigation with saline solution ( $\mathrm{NaCl} 0.9 \%)$ and suction. Intra-osseous implantation was performed as recommended by the ISO 10993-6 standard (2007) with the cylindrical parts of the samples inserted in the cancellous bone. Once implanted, the samples were in contact with bone along the sides while the bottom was facing a cavity in the bone. This enabled a study of how bone is formed around the implants under different conditions. The animals were treated and kept according to the conditions conformed by the EU requirements of farm animals (EC Directive 86/609). During the observation period, penicillin procaine and penicillin benzathine (Duplocilline ${ }^{\circledR}$, Intervet) were continued on days 3, 6 and 9 following surgery. The analgesic (flunixine, Meflosyl ${ }^{\circledR}$, Fort Dodge Santé Animale) was administered every other day for one week following surgery. After a 6-week implantation period, the animals were sacrificed by lethal injection of a barbiturate (Dolethal $^{\mathrm{ND}}$, Vetoquinol) and implanted sites were sampled.

The protocol for the study was approved by BIOMATECH (France) Ethical Committee on October 14, 2008.

\subsection{Sample Preparation}

The retrieved samples were dehydrated in ethanol solutions of increasing concentration, cleared in xylene, and embedded in poly-methyl methacrylate (PMMA). One longitudinal section in the long axis of the implant was obtained per specimen where one half of the sample was sectioned by a microcutting and grinding technique adapted from Donath et al [20]. The remaining half from each specimen was sputtered with a thin gold/palladium layer to enable SEM analysis.

\subsection{Histology}

Sections were stained with modified Paragon for semiquantitative and qualitative analyses. Histological analyses were performed using a Nikon microscope coupled with a digital camera (magnifications of x4, x10, x20 and $\mathrm{x} 40$ ). Semi-quantitative evaluation of local tolerance was performed according to ISO 10993-6 standard.

\subsection{Bone/Implant Contact and Adjacent Bone Density}

The samples prepared for SEM analysis were analyzed in backscatter mode using $15 \mathrm{keV}$ acceleration voltage (Leo 1550 FEG Gemini, Zeiss). The bone/implant contact was calculated from the images by measuring the contact length along the periphery of the sides and bottom of the implants, see example in Figure 2. Quantitative analysis was performed on the SEM images using a software (LeicaQwin) to calculate the bone density within the nearest $200 \mu \mathrm{m}$ of tissue surrounding the implants. Areas of soft tissue and bone along the sides and bottom of implants were determined from the difference in contrast between the two areas. The bone/soft tissue ratio was calculated from these measurements.

\section{Results and Discussion}

No local signs of necrosis were observed macroscopically with either the test or control samples. Moderate signs of hemorrhage were observed at the level of the soft tissues surrounding the type 1 and type 2 samples. No signs of exudates were observed with either the test or control samples with exception of one animal, which showed the presence of a subcutaneous liquid pocket in the right and left femur graded as marked exudates and increasing the mean grade of exudation similarly in all groups.

No local intolerance signs were observed for any of the samples except for one of the test 3 samples, where a marked infiltration of lymphocytes without significant implication on the bone healing was observed. Marked grade of newly formed bone showing characteristics of a mixture of lamellar and woven bone with osseous condensation were seen along the surface for all sample types. In terms of healing performance, the histology analysis demonstrated that the control samples and the

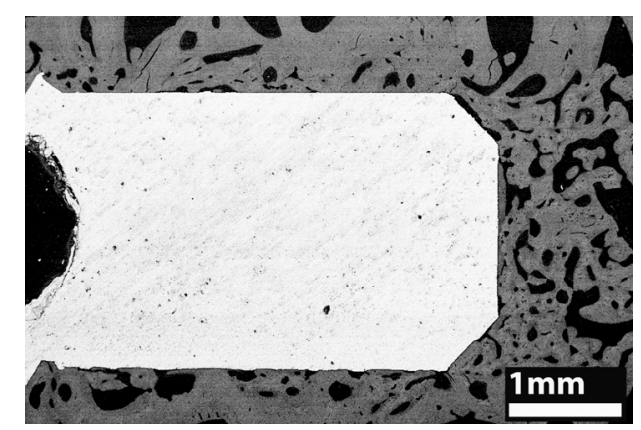

Figure 2. SEM-image of implanted sample (test 3). The image shows bone around the entire sample. 
test 1 and test 3 samples yielded marked and similar amount of newly formed bone and level of implant osseointegration. Sites implanted with test 2 samples showed inconsistent outcome with a slightly lower healing performance as compared to the previous samples. Very few osteoclasts were observed in the vicinity of the test 3 samples compared to moderate numbers for the other types of implants. Figure 3 shows active bone deposition on a test 1 sample.

The results from the bone/implant contact measurements are presented in Figure $\mathbf{4}$ as the average difference in contact between the test samples and the control sample in respective sheep. The displayed results are based on three values as the highest and lowest values were excluded from the calculations. Notably is the large impact of BMP-2 seen at the bottom of the samples. Here, the implants were not in contact with bone at implantation and the BMP-2 clearly induces more bone formation along the periphery of the implants at these conditions. At the sides of the implant, the results for the test samples were equivalent to the control samples and no significant difference could be seen. The apposition of new bone around the samples was not improved by the BHA coating as the control samples were equally integrated in the bone as the test 1 samples with an average bone contact of $62 \%$ on the sides and $52 \%$ at the bottom. The BMP-2 had no detectable effect along the sides of the implants. Here, the implants were in contact with bone immediately after implantation and the different conditions for healing and bone formation clearly affected the impact of the delivered BMP-2. The test 2 samples were the only ones with more bone apposition at the bottom compared to the sides with about $20 \%$ more bone contact at the bottom. Also notably is that the BIS did not affect the bone contact.

Figure 5 displays the results from the bone density measurements where the results are presented as the

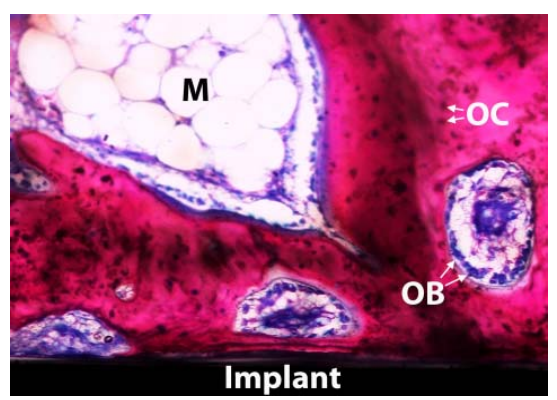

Figure 3. Histologic image $(\times 10)$ of active bone deposition around an implant with BHA coating. The black area in the lower part of the image is the implant, red color indicates osseous tissues with osteocytes (OC), the blue linings surrounding the bone are composed of active osteoblasts (OB) and white areas indicate bone marrow $(\mathrm{M})$. The sample was stained with modified Paragon. average difference in bone density between the test samples and the control sample in respective sheep. The results are based on three values as the highest and lowest values were excluded from the calculations. Here, it can be seen that the delivered BIS had a significant impact on the bone formation around the test 3 samples. This was also confirmed by the histology analysis where very slight numbers of osteoclasts were observed in the vicinity of these implants.

The greatest impact was seen at the bottom, which suggests that the delivered BIS was most effective in areas in total absence of bone. Notably is the lower bone density around the test 2 samples and this observation may find its explanation in the dual effect of BMP-2 that also activates apoptosis in postnatal osteoblasts [21,22]. BMP-2 has also been shown to decrease calcium deposition in vitro $[22,23]$ and the findings in this study suggest that the delivered BMP-2 actively promotes activation of newly recruited mesenchymal stem cells and osteoblast progenitor cells to produce bone directly at the HA coating while more mature osteoblasts already present in the bone at some distance from the implant are stimulated in a negative way. A higher concentration on BMP-2 in the
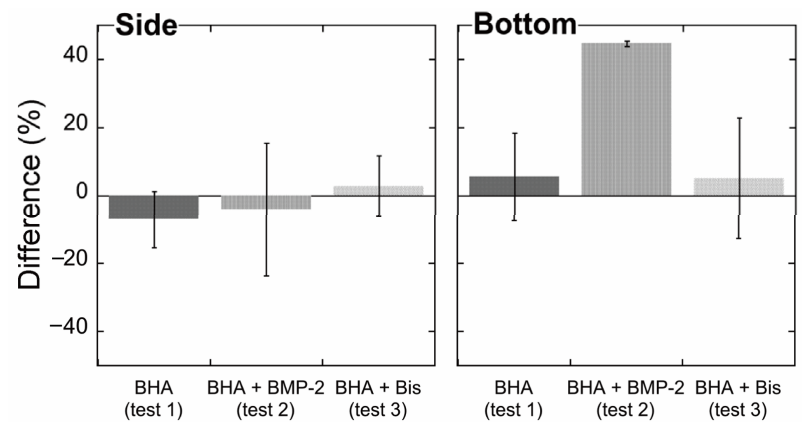

Figure 4. Average difference in bone/implant contact for the test samples compared to the control sample in respective sheep, error bars show the standard deviation for the three samples.
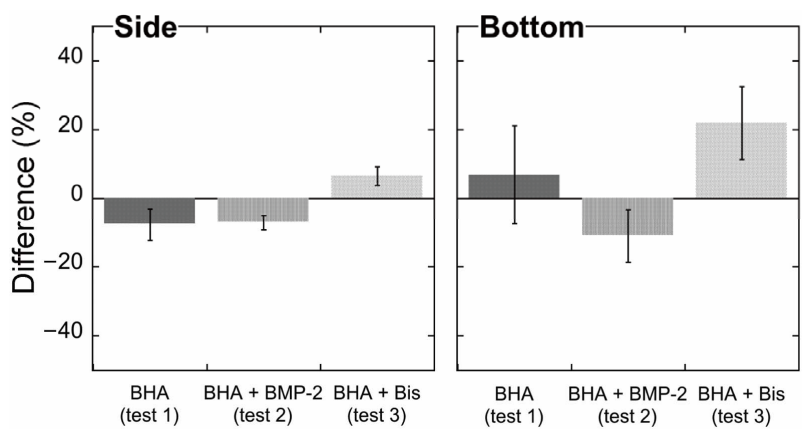

Figure 5. Average difference in adjacent bone density for the test samples compared to the control sample in respective sheep, error bars show the standard deviation for the three samples. 
loading solution might have rendered a more effective functionalization of the BHA coating, resulting in a higher amount of BMP-2 delivered to the tissues. This could have caused another biological response if the effect is dose-dependent. The BHA coating alone was not observed to have any impact on the bone formation around the implants after 6 weeks of implantation, which is in line with earlier studies [24]. Yet, it is in contrary to the positive effects of biomimetic HA on bone formation observed by Barrère et al [25].

The findings in this study prove that it is possible to obtain a positive effect on the bone formation in vivo via local delivery of active agents from implant coatings comprised of BHA. In the future, this strategy of incorporating active molecules to BHA coatings in the operating room immediately prior to implant insertion is suggested to be employed to deliver single drugs or combinations of drugs including, e.g., antibiotics from the surfaces to reduce the incident rate of surgery-related infections.

\section{Conclusions}

The study demonstrates an effective method to functionalize implants immediately prior to surgery. The functionalization procedure in the operating room did not lead to any infections in the animals and the active molecules delivered from the coatings were shown to have an impact on the bone formation. BMP-2 was shown to promote more bone formation directly along the periphery of the implants in areas without bone and BIS promoted more dense bone formation. The proposed strategy is also suggested to be used for local release of, e.g., antibiotics in the future to reduce the incident rate of surgery-related infections.

\section{Acknowledgements}

The Swedish Research Council is acknowledged for funding this study.

\section{REFERENCES}

[1] M. Dobzyniak, T. K. Fehring and S. Odum, "Early Failure in Total Hip Arthroplasty,” Clinical Orthopaedics and Related Research, Vol. 477, 2006, pp. 76-78. doi:10.1097/01.blo.0000203484.90711.52

[2] P. F. Sharkey, W. J. Hozack, R. H. Rothman, S. Shastri and S. M. Jacoby, "Why are Total Knee Arthroplasties Failing Today," Clinical Orthopaedics and Related Research, Vol. 404, 2002, pp. 7-13. doi:10.1097/00003086-200211000-00003

[3] S. Kurtz, K. Ong, E. Lau, F. Mowat and M. Halpern, "Projections of Primary and Revision Hip and Knee Arthroplasty in the United States from 2005 to 2030," Journal of Bone and Joint Surgery(American Volume), Vol. 89A, No. 4, 2007, pp. 780-785. doi:10.2106/JBJS.F.00222

[4] K. A. Krackow and D. S. Hungerford, "Revision and
Primary Hip and Knee Arthroplasty-A Cost-Analysis,” Clinical Orthopaedics and Related Research, Vol. 311, 1995, pp. 136-141.

[5] T. K. Fehring, S. Odum, W. L. Griffin, J. B. Mason and M. Nadaud, "Early Failures in Total Knee Arthroplasty," Clinical Orthopaedics and Related Research, Vol. 392, 2001, pp. 315-318. doi:10.1097/00003086-200111000-00041

[6] A. J. Mangram, T. C. Horan, M. L. Pearson, L. C. Silver and W. R. Jarvis, "Guideline for Prevention of Surgical Site Infection, 1999," Infection Control and Hospital Epidemiology, Vol. 20, No. 4, 1999, pp. 250-278. doi:10.1086/501620

[7] L. Y. Carreon, R. M. Puno, J. R. Dimar, S. D. Glassman and J. R. Johnson, "Perioperative Complications of Posterior Lumbar de Compression and Arthrodesis in Older Adults," Journal of Bone and Joint Surgery(American Volume), Vol. 85A, No. 11, 2003, pp. 2089-2092.

[8] L. Pulido, E. Ghanem, A. Joshi, J. J. Purtill and J. Parvizi, "Periprosthetic Joint Infection: The Incidence, Timing, and Predisposing Factors," Clinical Orthopaedics and Related Research, Vol. 466, No. 7, 2008, pp. 1710-1715. doi:10.1007/s11999-008-0209-4

[9] A. G. Gristina, "Implant Failure and the Immune-Incom petent Fibro-Inflammatory Zone,” Clinical Orthopaedics and Related Research, Vol. 298, 1994, pp. 106-118.

[10] D. Campoccia, L. Montanaro and C. R. Arciola "The Significance of Infection Related to Orthopedic Devices and Issues of Antibiotic Resistance,” Biomaterials, Vol. 27, No. 11, 2006, pp. 2331-2339. doi:10.1016/j.biomaterials.2005.11.044

[11] I. Landor, P. Vavrik, A. Sosna, D. Jahoda, H. Hahn and M. Daniel, "Hydroxyapatite Porous Coating and the Osteointegration of the Total Hip Replacement," Archives of Rthopaedic and Trauma Surgery, Vol. 127, No. 2, 2007, pp. 81-89. doi:10.1007/s00402-006-0235-1

[12] R. G. T. Geesink, "Osteoconductive Coatings for Total Joint Arthroplasty,” Clinical Orthopaedics and Related Research, Vol. 395, 2002, pp. 53-65. doi:10.1097/00003086-200202000-00007

[13] R. Z. LeGeros, J. P. LeGeros Hydroxyapatite and T. Kokubo, "Bioceramics and Their Clinical Applications," Woodhead Publishing Limited, Sawston, 2008.

[14] U. Brohede, J. Forsgren, S. Roos, A. Mihranyan, H. Engqvist and M. Strømme, "Multifunctional Implant Coatings Providing Possibilities for Fast Antibiotics Loading with Subsequent Slow Release," Journal of Materials Science-Materials in Medicine, Vol. 20, No. 9, 2009, pp. 1859-1867. doi:10.1007/s10856-009-3749-6

[15] J. Åberg, U. Brohede, A. Mihranyan, M. Strømme and H. Engqvist, "Bisphosphonate Incorporation in Surgical Implant Coatings by Fast Loading and Co-Precipitation at Low Drug Concentrations,” Journal of Materials Science: Materials in Medicine, Vol. 20, No. 10, 2009, pp. 20532061.

[16] S. Piskounova, J. Forsgren, U. Brohede, H. Engqvist and M. Strømme, "In Vitro Characterization of Bioactive Titanium Dioxide/Hydroxyapatite Surfaces Functionalized with BMP-2,” Journal of Biomedical Materials Research Part B-Applied Biomaterials, Vol. 91B, No. 2, 2009, pp. 780787. doi:10.1002/jbm.b.31456 
[17] U. Brohede, S. X. Zhao, F. Lindberg, A. Mihranyan, J. Forsgren, M. Strømme, et al., "A Novel Graded Bioactive High Adhesion Implant Coating,” Applied Surface Science, Vol. 255, No. 17, 2009, pp. 7723-7728.

doi:10.1016/j.apsusc.2009.04.149

[18] J. Forsgren, F. Svahn, T. Jarmar and H. Engqvist, "Formationand Adhesion of Biomimetic Hydroxyapatite Deposited on Titanium Substrates,” Acta Biomaterialia, Vol. 3, No. 6, 2007, pp. 980-984. doi:10.1016/j.actbio.2007.03.006

[19] A. Mihranyan, J. Forsgren, M. Strømme and H. Engqvist, "Assessing Surface Area Evolution during Biomimetic Growth of Hydroxyapatite Coatings,” Langmuir, Vol. 25, No. 3, 2009, pp. 1292-1295. doi:10.1021/la803520k

[20] K. Donath and G. Breuner, "A Method for the Study of Undecalcified Bones and Teeth with Attached Soft-Tiss ues-the Sage-Schliff (Sawing and Grinding) Technique," Journal of Oral Pathology and Medicine, Vol. 11, No. 4, 1982, pp. 318-326. doi:10.1111/j.1600-0714.1982.tb00172.x

[21] E. Hay, J. Lemonnier, O. Fromigue and P. J. Marie, "Bone Morphogenetic Protein-2 Promotes Osteoblast Apoptosis through a Smad-Independent, Protein Kinase C-Dependent Signaling Pathway,” Journal of Biological Chemistry, Vol. 276, No. 31, 2001, pp. 29028-29036.
doi:10.1074/jbc.M011265200

[22] E. Hay, J. Lemonnier, O. Fromigue, H. Guenou and P. J. Marie, "Bone Morphogenetic Protein Receptor IB Signaling Mediates Apoptosis Independently of Differentiation in Osteoblastic Cells," Journal of Biological Chemistry, Vol. 279, No. 3, 2004, pp. 1650-1658.

[23] C. A. Luppen, E. Smith, L. Spevak, A. L. Boskey and B. Frenkel, "Bone Morphogenetic Protein-2 Restores Mineralization in Glucocorticoid-Inhibited MC3T3-E1 Os- teoblast Cultures,” Journal of Bone and Mineral Research, Vol. 18, No. 7, 2003, pp. 1186-1197. doi:10.1359/jbmr.2003.18.7.1186

[24] F. M. He, G. L. Yang, X. X. Wang and S. F. Zhao, "Bone Responses to Rough Titanium Implants Coated with Biomimetic Ca-P in Rabbit Tibia,” Journal of Biomedical Materials Research Part B-Applied Biomaterials, Vol. 90B, No. 2, 2009, pp. 857-863. doi:10.1002/jbm.b.31355

[25] F. Barrere, C. M. van der Valk, G. Meijer, R. A. J. Dalmeijer, K. de Groot and P. Layrolle, "Osteointegration of Biomimetic Apatite Coating Applied onto Dense and Porous Metal Implants in Femurs of Goats,” Journal of Biomedical Materials Research Part B-Applied Biomaterials, Vol. 67B, No. 1, 2003, pp. 655-665. doi:10.1002/jbm.b.10057 